\title{
Gold nanoparticles PVA thin film as Q-switcher in neodymium doped fiber laser cavity
}

\author{
Ahmad Haziq Aiman Rosol ${ }^{1,2}$, Kaharudin Dimyati ${ }^{1,2}$, Nur Farhanah Zulkipli ${ }^{2}$, Retno Apsari ${ }^{2}$, \\ Moh Yasin ${ }^{2}$, Sulaiman Wadi Harun ${ }^{1, a}$ \\ ${ }^{1}$ University of Malaya, 50603 Kuala Lumpur, Malaysia \\ ${ }^{2}$ Airlangga University, Surabaya, Indonesia \\ ${ }^{a}$ swharun@um.edu.my
}

Corresponding author: Sulaiman Wadi Harun, swharun@um.edu.my

PACS 42.82.Gw, 42.81.-i, 42.55.Wd

ABSTRACT A gold nanoparticle-based saturable absorber (SA) was successfully employed in Neodymiumdoped fiber laser (NDFL) cavity for pulse generation via a Q-switching mechanism for the first time. The SA device was made by mixing the gold nanoparticles into PVA solution before being dried to form a thin film was included into the NDFL cavity, which was optimized to obtain Q-switched pulses centered at wavelength of $1089 \mathrm{~nm}$. The pulse rate of the laser rose from 37.37 to $49.60 \mathrm{kHz}$ while the pulse width fell from 4.82 to $3.84 \mu \mathrm{s}$ as the $808 \mathrm{~nm}$ pump laser was raised from 98 to $144 \mathrm{~mW}$. At the highest input pump power of $144 \mathrm{~mW}$, the maximum output power and pulse energy was achieved at $0.52 \mathrm{~mW}$ and $10.48 \mathrm{~nJ}$, respectively. The developed Q-switched laser has numerous applications including, material micromachining, communication, sensing, etc.

KEYWORDS neodymium doped fiber laser, gold nanoparticles, plasmonic

ACKNOWLEDGEMENTS This work was supported by the Airlangga University Research Grant (2021) and the University of Malaya (Grant No ST036-2020)

For citation Rosol A.H.A., Dimyati K., Zulkipli N.F., Apsari R., Yasin M., Harun S.W. Gold nanoparticles PVA thin film as Q-switcher in neodymium doped fiber laser cavity. Nanosystems: Phys. Chem. Math., 2022, 13 (1), 50-55.

\section{Introduction}

Current Q-switched fiber lasers have received many researchers' attention, owing to their potential applications in numerous areas including telecommunication, material processing, medical diagnostics, spectroscopy, and optical sensing [1-3]. Conventionally, these lasers were realized using an active method employing external modulators [4]. In comparison with this method, the passive method using saturable absorber (SA) is less complicated while offering many advantages in terms of price, practicality, simplicity, and compactness [5]. In Q-switching, the SA is directly added into a laser cavity to generate short pulses due to the modulation of cavity loss and produce short pulses. When the laser intensity reaches the saturation power of the SA, the cavity loss is suddenly decreased, thus allowing more photons to be transmitted, leading to the production of a sequence of pulse train within the oscillator. To date, various nanomaterials such as single-wall carbon nanotubes [6], graphene [6], topological insulators [7,8], and transition metal dichalcogenides [9-12] have been investigated and reported as SA. These materials have common advantages of wide absorption, ultrafast response time, and large third-order nonlinearity [13]. For example, Niu et al. proposed the employment of tin disulfide $\left(\mathrm{SnS}_{2}\right)$, which has a bandgap value of $2.24 \mathrm{eV}$ as a Q-switcher. The laser successfully produced a short pulse with a maximum repetition rate of $233 \mathrm{kHz}$ and minimum pulse width of $0.51 \mu \mathrm{s}$ at $1532.7 \mathrm{~nm}$ [14].

Additionally, noble metal nanoparticles have also gained attention for SA applications owing to their close-lying energy bands, which allow a free electron to move. The energy filled the electron on the conduction band till it stimulated the plasmonic resonance to the surface as the photons interact with metal nanoparticles [15]. The metal nanoparticles could also be employed for pulse generation owing to the broadband absorption induced by the surface plasmon resonance and ultrafast response time [16-18]. For instance, Muhammad et al. demonstrated Q-switched erbium-doped fiber laser (EDFL) by using copper nanoparticles as saturable absorbers [19]. In another work, Ahmad et al. reported Q-switched pulse generation using gold nanoparticles obtained through thermal deposition method whereby maximum pulse width of $4.25 \mu \mathrm{s}$ was realized [20]. All the reported works are carried out in $1.55 \mu \mathrm{m}$ region based on the EDFLcavity.

More recently, interest in Q-switched neodymium-doped fiber laser (NDFL) operating in wavelength region around $1 \mu \mathrm{m}$ is also increasing. NDFL can produce lasing in $1060 \mathrm{~nm}$ wavelength region through four-level transition of ${ }^{4} \mathrm{~F}_{3 / 2} \rightarrow$ ${ }^{4} \mathrm{I}_{11 / 2}$. Until recently, most Q-switched fiber lasers at around $1060 \mathrm{~nm}$ were achieved using an ytterbium-doped fiber laser. In this paper, a Q-switched NDFL operating at $1060 \mathrm{~nm}$ was demonstrated using the gold nanoparticles (GNPs) as SA.

(C) Rosol A.H.A., Dimyati K., Zulkipli N.F., Apsari R., Yasin M., Harun S.W., 2022 
The SA was fabricated by embedding the GNPs into the PVA by drop-casting method to form a SA thin film. This was then added into the NDFL cavity to modulate loss for generation of short pulse.

\section{SA fabrication and characterizations}

Figure 1 shows the fabrication process flow of GNPs based thin film using sodium borohydride $\left(\mathrm{NaBH}_{4}\right)$, gold (III) chloride trihydrate $\left(\mathrm{HAuCl}_{4} \cdot 3 \mathrm{H}_{2} \mathrm{O}\right)$, poly (sodium 4-styrenesulfonate) (PSSS), and tri-sodium citrate (TSC) liquids. All chemicals and solvents were used as received without further purification. At first, the GNPs were made using a $\mathrm{NaBH}_{4}$ reduction method. $3 \mathrm{~mL}$ of $\mathrm{NaBH}_{4}, 3 \mathrm{~mL}$ PSSS and $50 \mathrm{~mL}$ TSC were poured into a beaker containing $1000 \mathrm{~mL}$ deionized water (with the resistivity of $18 \mathrm{M} \Omega$ ) while stirring at $450 \mathrm{rpm}$. Then, $50 \mathrm{~mL} \mathrm{HAuCl}_{4}(5 \mathrm{mM}$, in water) was slowly dropped into the mixture in a rate of $\sim 2 \mathrm{~mL} / \mathrm{min}$ with continuous stirring followed by the addition of the excess amount of TSC $(20 \mathrm{~mL})$. After that the mixture was left for 5 minutes to allow the reaction among the chemicals to complete before it was continued with a centrifugation process for cleaning purposes. We mixed the prepared GNPs solution with PVA liquid to prepare a SA thin film. The PVA liquid was obtained by dissolving PVA powder into distilled water. The powder was completely dissolved after a thorough stirring process at high temperature of $145^{\circ} \mathrm{C}$. The GNPs PVA mixture was then slowly stirred for about 2 hours to obtain an homogenous suspension solution, which was then used to prepare the thin film. It was poured and spread onto a petri dish before it was left to dry at room temperature for about 48 hours. The dried thin film was peeled so that it can be integrated into the laser cavity for the Q-switching experiment.

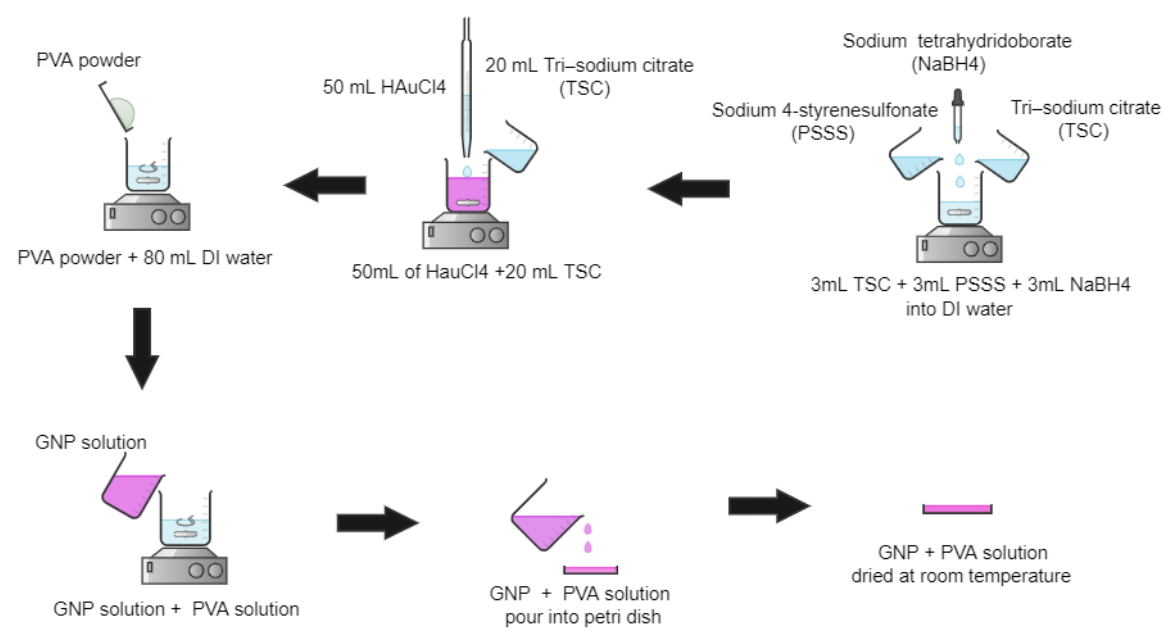

FIG. 1. Fabrication step for GNPs thin film

The non-linear absorption of the thin film was then investigated using a balanced twin-detector measurement technique. The light pulses from a homemade mode-locked fiber laser operating at pulse width of 1.3 ps and repetition rate of $2.5 \mathrm{MHz}$ was used in the measurement. The non-linear absorption curve was obtained by comparing the peak power of the laser before and after the thin film. As shown in Fig. 2(a), the absorption reduces with the increase of input optical intensity. This indicates that the fabricated SA film has an excellent saturable absorption property, which could be used for pulse generation. The modulation depth, non-saturable absorption and saturable intensity of the film are obtained as $13.6 \%, 47.2 \%$, and $0.5 \mathrm{MW} / \mathrm{cm}^{2}$ respectively. It is worth noting that we have not observed any nonlinear response from pure PVA film, confirming that the saturable absorption property was solely originated from the GNPs. The linear optical absorption profile was also examined for this SA using a white light source and optical spectrum analyzer (OSA). Fig. 2(b) shows the absorbance spectrum of the GNPs PVA film, which was measured within a wavelength range from 1000 to $1300 \mathrm{~nm}$. As shown in the figure, the absorption of about $5 \mathrm{~dB}$ was obtained at wavelength of $1080 \mathrm{~nm}$.

\section{Cavity configuration}

The schematic diagram of the proposed laser setup is illustrated in Fig. 3. It used the newly developed GNPs PVA thin film as Q-switcher. An $11 \mathrm{~m}$ long Nd-doped fiber (NDF) with a peak absorption of $8.5 \mathrm{~dB} / \mathrm{m}$ at $810 \mathrm{~nm}$ was employed as the laser active medium for the operation at $1080 \mathrm{~nm}$ region. The Nd-doped active fiber has a numerical aperture of 0.18 and a cutoff wavelength of $990 \mathrm{~nm}$. We employed a single mode fiber (SMF) coupled $808 \mathrm{~nm}$ laser diode with the maximum power of $\sim 250 \mathrm{~mW}$ as the pump source. The SMF has a cutoff wavelength of $780 \mathrm{~nm}$ and core/clad diameter of $4.4 / 125 \mu \mathrm{m}$. The pump light was launched into the ring NDFL cavity via an 808/1064 wavelength division multiplexer. A polarization-insensitive isolator is incorporated into the NDFL cavity to provide unidirectional operation. An 80/20 optical coupler is employed to extract $20 \%$ of the generated laser for measurement. The total cavity length 
(a)
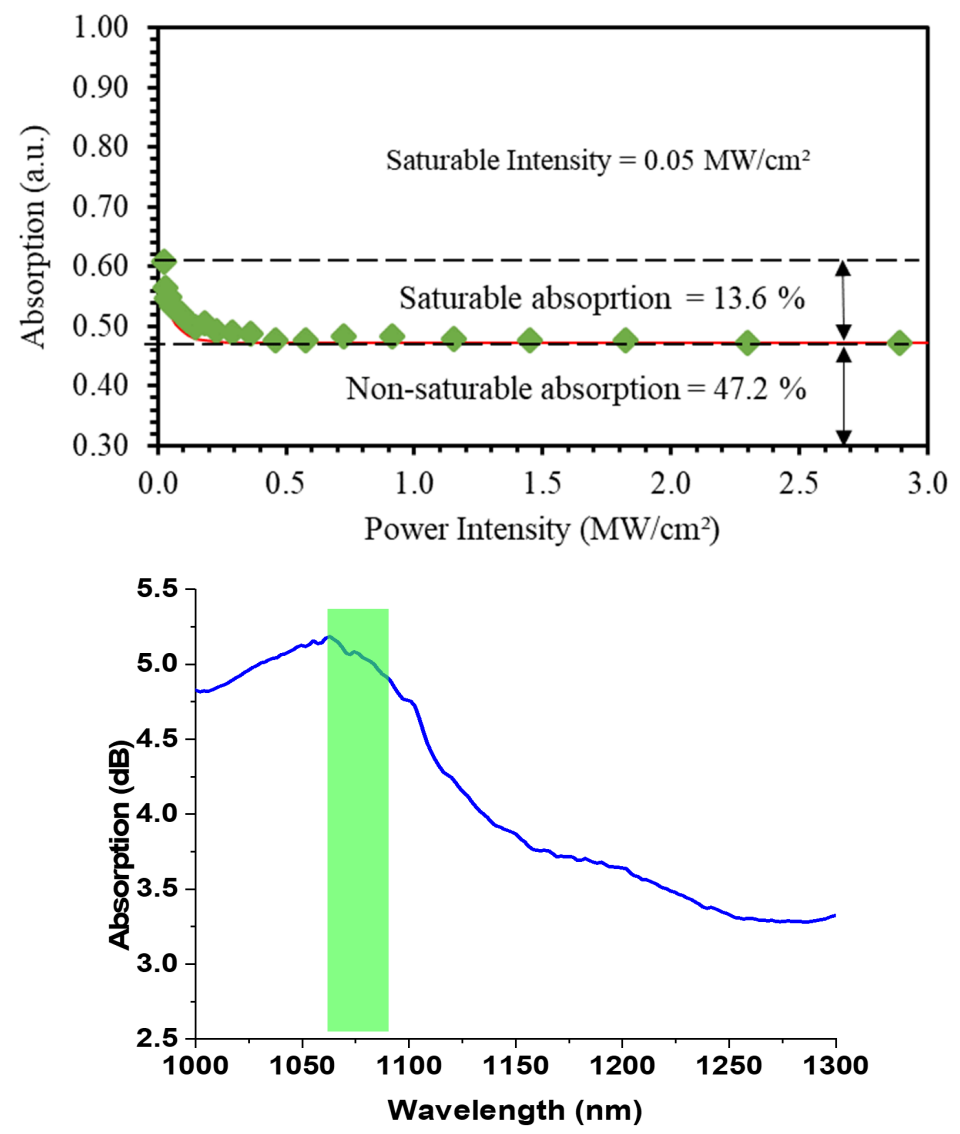

FIG. 2. (a) The non-linear and (b) linear absorption properties of GNPs thin film

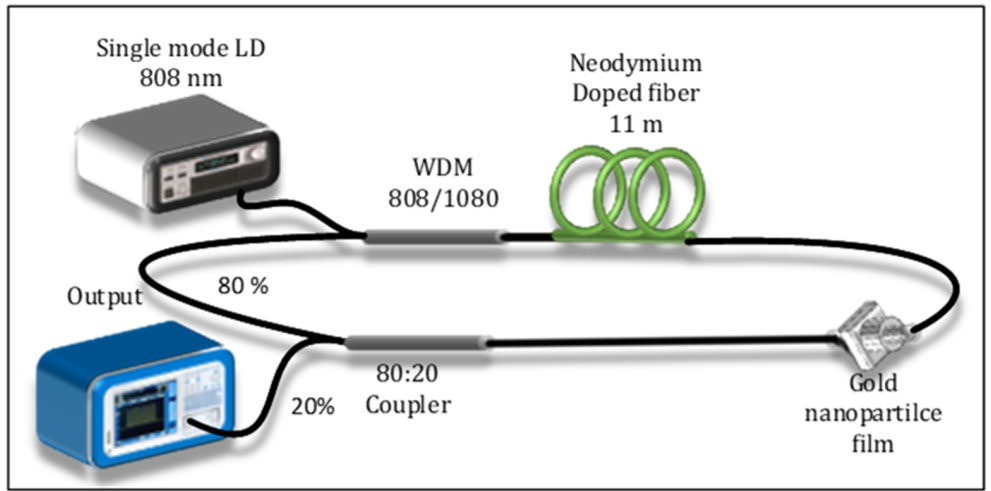

FIG. 3. The illustration of NDFL configuration

was about $13.5 \mathrm{~m}$. The output pulses of the passively Q-switched fiber laser were characterized by a fast-speed InGaAs photodetector, $350 \mathrm{MHz}$ digital oscilloscope (GWINSTEK, GDS-3352), 7.8 GHz spectrum analyzer (Anritsu), power meter (PM100D-S122C), and optical spectrum analyzer (OSA, AQ6317).

\section{Result and discussion}

In this experiment, a self-starting Q-switched pulse train was realized as the pump power was raised above the threshold of $98 \mathrm{~mW}$. The output pulse train of the laser was presented for three different pump powers of 98,121 and $144 \mathrm{~mW}$ as shown in Figs. 4(a,b and c), respectively. The pulse train has a peak-to-peak spacing (or pulse period) of $26.75,22.53$ and $20.16 \mu$ s, which corresponds to a repetition rate of $37.37,44.38$ and $49.6 \mathrm{kHz}$, respectively. The pulse period reduces with the pump power due to the Q-switching effect, which is dependent on the saturation of the SA. An increase of pump intensity improves the gain in the cavity and thus it saturates the SA within a shorter time. This leads to faster bleaching of the GNPs SA, resulting in the reduction of the pulse period. This is a typical trend for a passively Q-switched lasers. 

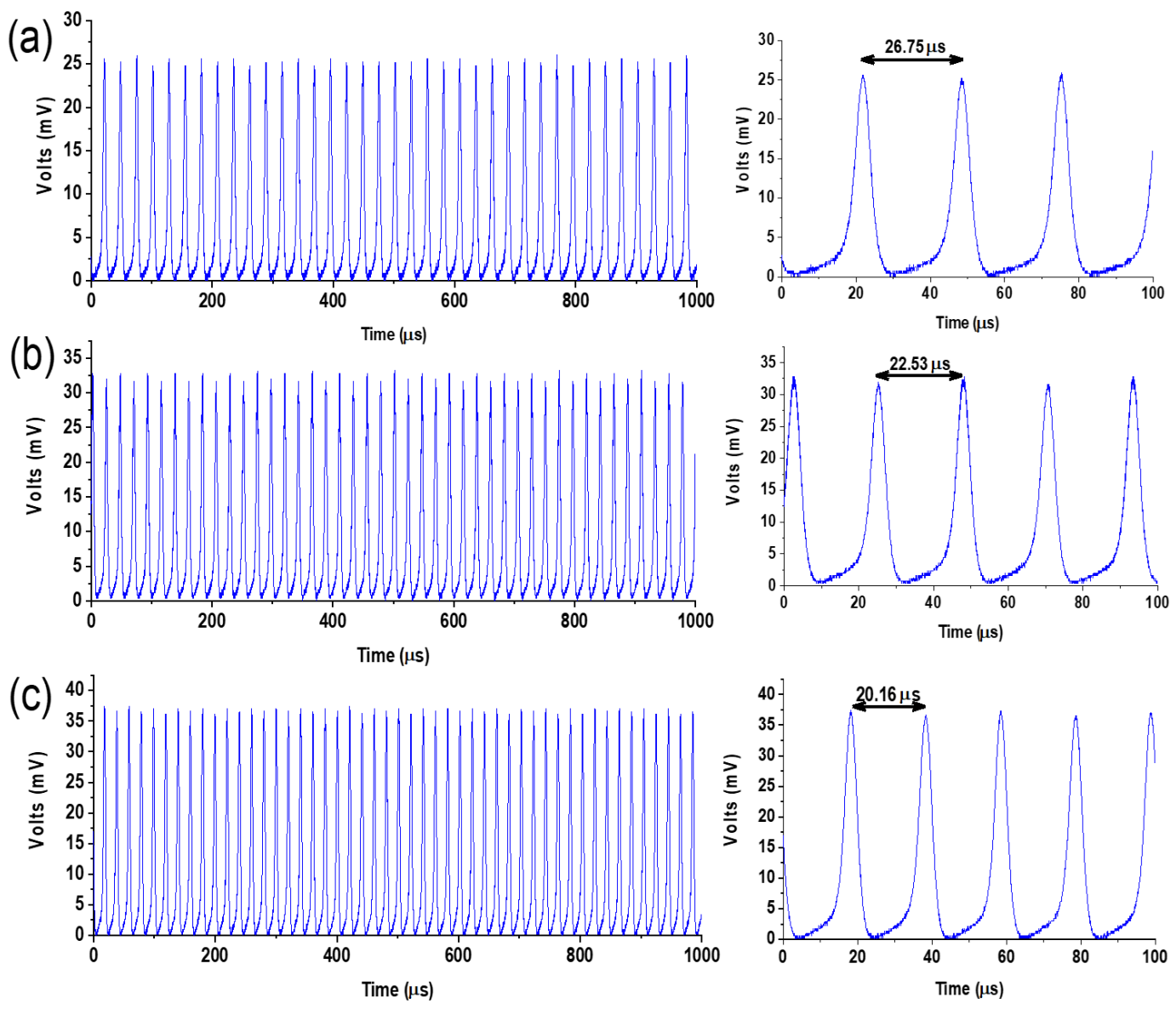

FIG. 4. The pulse train of the Q-switched NDFL with AuNPs PVA film-based SA at three different pump powers of (a) $98 \mathrm{~mW}$; (b) $121 \mathrm{~mW}$ and (c) $144 \mathrm{~mW}$

Figure 5(a) displays the typical output spectral characteristic of the Q-switched NDFL at the pump power of $98 \mathrm{~mW}$. As shown in the figure, the central wavelength of the Q-switched operation is at approximately 1089 nm which is due to $\mathrm{Nd}$ ions transition from ${ }^{4} \mathrm{~F}_{3 / 2}$ to ${ }^{4} \mathrm{~F}_{11 / 2}$ in four level laser system. Fig. 5(b) shows the RF spectrum of the pulse train at $144 \mathrm{~mW}$ pump power. The fundamental frequency was recorded at $49.6 \mathrm{kHz}$ with a SNR of $50 \mathrm{~dB}$, indicating the stability of the Q-switched laser. As the pump power was increased, the rise time and fall time of the Q-switched pulse become concurrently shorter [21] and this leads to the increase of repetition rate. As shown in Fig. 5(c), the repetition rate varied from 37.37 to $49.60 \mathrm{kHz}$ and the pulse width changed from 4.82 to $3.84 \mu \mathrm{s}$ as the applied pump power increased from 98 to $144 \mathrm{~mW}$. However, the pulse train was unstable when the applied pump power was greater than $144 \mathrm{~mW}$. This is most probably due to the rapid bleaching of the AuNPs SA under the high pump power. As shown in Fig. 5(d), the variation of average output power, single pulse energy and peak power against the pump power shows a generally rising trend. The maximum output power, pulse energy and peak power was achieved at $0.52 \mathrm{~mW}, 10.48 \mathrm{~nJ}$ and $2.73 \mathrm{~mW}$ at the highest input pump power of $144 \mathrm{~mW}$. It is worthy to note that the GNP SA could also be used to generate mode-locked pulses operating in the picosecond regime. However, a slight modification of the NDFL cavity is required to enhance the nonlinear effect as well as to balance the group velocity dispersion.

\section{Conclusion}

A Q-switched laser has been successfully shown in NDFL cavity using the newly developed GNPs PVA thin film as a SA device. The Q-switched pulses were successfully produced, and they operate at central wavelength of $1089 \mathrm{~nm}$ with maximum repetition rate of $49.6 \mathrm{kHz}$. The minimum pulse width, maximum pulse energy and maximum peak power of the Q-switched NDFL were obtained at about $3.84 \mu \mathrm{s}, 10 \mathrm{~nJ}$ and $2.75 \mathrm{~mW}$ respectively. The experimental results suggest that GNPs are a favorable material for Q-switching application in 1-micron region. 


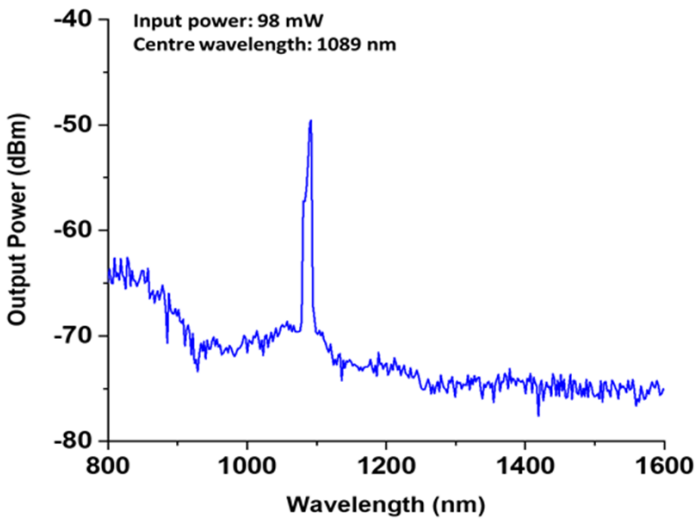

(a)

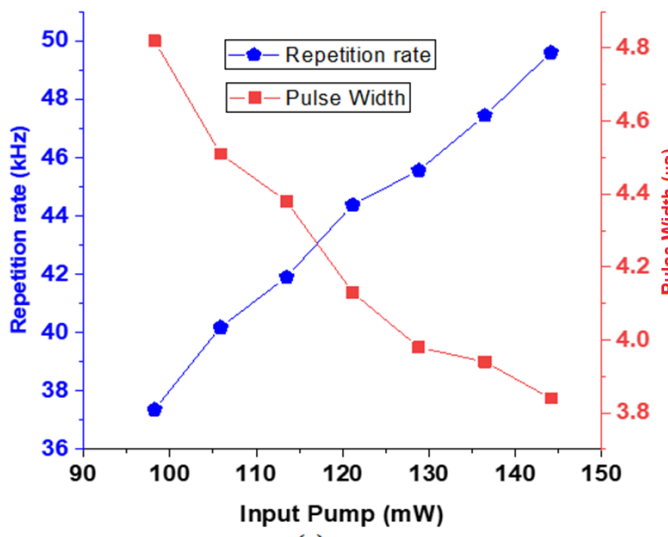

(c)

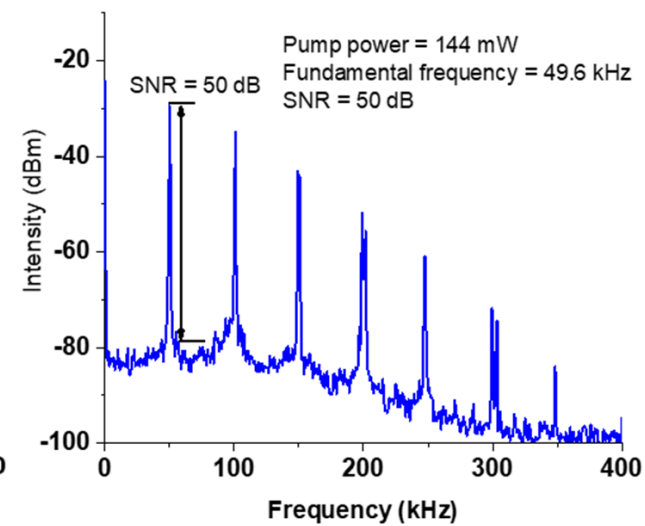

(b)

FIG. 5. The output pulse characteristics (a) Optical wavelength spectrum (b) RF spectrum (c) repetition rate and pulse width versus pump power and (d) output power, pulse energy and peak power against pump power

\section{References}

[1] Wang X., Cui Y., et al. Recent advances in the functional 2D photonic and optoelectronic devices. Advanced Optical Materials, 2019,7 , 1801274.

[2] Yefet S., Pe'er A. A review of cavity design for Kerr lens mode-locked solid-state lasers. Applied Sciences, 2013,3 , P. 694-724.

[3] Fermann M.E., Hartl I. Ultrafast fiber laser technology. IEEE J. of Selected Topics in Quantum Electronics, 2009, 15, P. 191-206.

[4] Jeon M.-Y., Lee H.K., et al. Harmonically mode-locked fiber laser with an acousto-optic modulator in a Sagnac loop and Faraday rotating mirror cavity. Optics Communications, 1998, 149, P. 312-316.

[5] Wang X.-D., Luo Z.-C., et al. Microfiber-based gold nanorods as saturable absorber for femtosecond pulse generation in a fiber laser. Applied Physics Letters, 2014, 105, 161107.

[6] Li X., Wu K., et al. Single-wall carbon nanotubes and graphene oxide-based saturable absorbers for low phase noise mode-locked fiber lasers. Scientific Reports, 2016, 6, 25266.

[7] Luo Z.-C., Liu M., et al. $2 \mathrm{GHz}$ passively harmonic mode-locked fiber laser by a microfiber-based topological insulator saturable absorber. Optics letters, 2013, 38, P. 5212-5215.

[8] Haris H., Harun S., et al. Passively Q-switched Erbium-doped and Ytterbium-doped fibre lasers with topological insulator bismuth selenide $\left(\mathrm{Bi}_{2} \mathrm{Se}_{3}\right)$ as saturable absorber. Optics \& Laser Technology, 2017, 88, P. 121-127.

[9] Xia H., Li H., et al. Ultrafast erbium-doped fiber laser mode-locked by a CVD-grown molybdenum disulfide (MoS 2) saturable absorber. Optics Express, 2014, 22, P. 17341-17348.

[10] Yan P., Liu A., et al. Passively mode-locked fiber laser by a cell-type WS 2 nanosheets saturable absorber. Scientific Reports, 2015,5 , 12587.

[11] Niu K., Sun R., et al Passively mode-locked Er-doped fiber laser based on SnS 2 nanosheets as a saturable absorber. Photonics Research, 2018, 6, P. 72-76.

[12] Zhang H., Ma P., et al. Palladium selenide as a broadband saturable absorber for ultra-fast photonics. Nanophotonics, 2020,9 (8), P. $2557-2567$.

[13] Sobon G. Mode-locking of fiber lasers using novel two-dimensional nanomaterials: graphene and topological insulators. Photonics Research, 2015, 3, P. A56-A63.

[14] Niu K., Chen Q., et al. Passively Q-switched erbium-doped fiber laser based on SnS 2 saturable absorber. Optical Materials Express, 2017, 7, P. 3934-3943.

[15] Gurudas U., Brooks E., et al. Saturable and reverse saturable absorption in silver nanodots at $532 \mathrm{~nm}$ using picosecond laser pulses. J. of Applied Physics, 2008, 104, 073107.

[16] Kang Z., Xu Y., et al. Passively mode-locking induced by gold nanorods in erbium-doped fiber lasers. Applied Physics Letters, $2013,103,041105$.

[17] Jiang T., Xu Y., et al Passively Q-switching induced by gold nanocrystals. Applied Physics Letters, $2012,101,151122$.

[18] Kang Z., Guo X., et al. Gold nanorods as saturable absorbers for all-fiber passively Q-switched erbium-doped fiber laser. Optical Materials Express, 2013, 3, P. 1986-1991. 
[19] Muhammad A., Ahmad M., et al. Q-switching pulse operation in 1.5- $\mu$ m region using copper nanoparticles as saturable absorber. Chinese Phys. Lett., 2017, 34, 034205 .

[20] Ahmad M., Muhammad A., et al. Gold nanoparticle based saturable absorber for Q-switching in $1.5 \mu$ m laser application. Laser Phys., 2017, 27, 115101.

[21] Chen Y., Zhao C., et al. Large energy, wavelength widely tunable, topological insulator Q-switched erbium-doped fiber laser. IEEE J. of Selected Topics in Quantum Electronics, 2013, 20, P. 315-322.

Submitted 2 December 2021; revised 12 December 2021; accepted 13 December 2021

Information about the authors:

Ahmad Haziq Aiman Rosol - Department of Electrical Engineering, University of Malaya, 50603 Kuala Lumpur, Malaysia; Department of Engineering, Faculty of Advanced Technology and Multidiscipline, Airlangga University, Surabaya, Indonesia; haziqaiman1992@gmail.com

Kaharudin Dimyati - Department of Electrical Engineering, University of Malaya, 50603 Kuala Lumpur, Malaysia; Department of Engineering, Faculty of Advanced Technology and Multidiscipline, Airlangga University, Surabaya, Indonesia; kaharudin@um.edu.my

Nur Farhanah Zulkipli - Department of Physics, Faculty of Science and Technology, Airlangga University, Surabaya, Indonesia; nurfarhanahzulkipli@yahoo.com

Retno Apsari - Department of Physics, Faculty of Science and Technology, Airlangga University, Surabaya, Indonesia; retna-a@fst.unair.ac.id

Moh Yasin - Department of Physics, Faculty of Science and Technology, Airlangga University, Surabaya, Indonesia; yasin@fst.unair.ac.id

Sulaiman Wadi Harun - Department of Electrical Engineering, University of Malaya, 50603 Kuala Lumpur, Malaysia; swharun@um.edu.my

Conflict of interest: the authors declare no conflict of interest. 